Article

\title{
Four-Dimensional Almost Einstein Manifolds with Skew-Circulant Structures
}

\author{
Iva Dokuzova ${ }^{1, \ddagger, *(\mathbb{D})}$ and Dimitar Razpopov ${ }^{2}$ (D)
}

1 Department of Algebra and Geometry, Faculty of Mathematics and Informatics, University of Plovdiv Paisii Hilendarski, Plovdiv, Bulgaria, dokuzova@uni-plovdiv.bg

2 Department of Mathematics and Informatics, Faculty of Economics, Agricultural University of Plovdiv, Bulgaria, razpopov@au-plovdiv.bg

* Correspondence: dokuzova@uni-plovdiv.bg; Tel.: 359887977638

$\ddagger \quad$ These authors contributed equally to this work.

\begin{abstract}
We consider a four-dimensional Riemannian manifold $M$ equipped with an additional tensor structure $S$, whose fourth power is minus identity and the second power is an almost complex structure. In a local coordinate system the components of the metric $g$ and the structure $S$ form skew-circulant matrices. Both structures $S$ and $g$ are compatible, such that an isometry is induced in every tangent space of $M$. By a special identity for the curvature tensor, generated by the Riemannian connection of $g$, we determine classes of an Einstein manifolds and an almost Einstein manifolds. For such manifolds we obtain propositions for the sectional curvatures of some special 2-planes in a tangent space of $M$. We consider an almost Hermitian manifold associated with the studied manifold and find conditions for $g$, under which it is a Kähler m anifold. We construct some examples of the considered manifolds on Lie groups.
\end{abstract}

Keywords: Riemannian manifold; Einstein manifold; sectional curvatures; Ricci curvature; Lie group

MSC: 53B20; 53C15; 53C25; 53C55; 22E60

\section{Introduction}

The right circulant matrices and the right skew-circulant matrices are Toeplitz matrices, which are thoroughly studied in [1] and [3]. The set of invertible circulant (skew-circulant) matrices form a group with respect to the matrix multiplication. Such matrices have application to geometry, linear codes, graph theory, vibration analysis (for example [2,7,9,11-13]).

A. Gray, L. Hervella and L. Vanhecke used curvature identities to classify and to study the almost Hermitian manifolds (for instance in $[4-6,15]$ ). The Hermitian manifolds form a class of manifolds with an integrable complex structure $J$. The class of the Kähler manifolds is their subclass and such manifolds have a parallel structure $J$. According to A. Gray, the Kähler manifolds have an especially rich geometric structure, due to the Kähler curvature identity $R(\cdot, \cdot, J \cdot, J \cdot)=R(\cdot, \cdot, \cdot, \cdot)$. Some of the recent investigations on the curvature properties of the almost Hermitian manifolds are made in $[8,10,14,16]$.

In the present work we study a four-dimensional differentiable manifold $M$ with a Riemannian metric $g$. The manifold $M$ is equipped with an additional tensor structure $S$ of type $(1,1)$, which satisfies $S^{4}=-\mathrm{id}$. Moreover, the component matrix of $S$ is a special skew-circulant matrix. The structure $S$ is compatible with $g$, such that an isometry is induced in every tangent space of $M$. Such 


\section{Preliminaries}

Let $M$ be a 4-dimensional Riemannian manifold equipped with a tensor structure $S$ in every tangent space $T_{p} M$ at a point $p$ on $M$. Let $S$ have a skew-circulant matrix, with respect to some basis $\left\{e_{i}\right\}$, as follows

$$
\left(S_{j}^{k}\right)=\left(\begin{array}{cccc}
0 & 1 & 0 & 0 \\
0 & 0 & 1 & 0 \\
0 & 0 & 0 & 1 \\
-1 & 0 & 0 & 0
\end{array}\right)
$$

Then $S$ has the property

$$
S^{4}=-\mathrm{id}
$$

Let the metric $g$ and the structure $S$ satisfy

$$
g(S x, S y)=g(x, y)
$$

Here and anywhere in this work, $x, y, z, u$ will stand for arbitrary elements of the algebra on smooth vector fields on $M$ or vectors in $T_{p} M$. The Einstein summation convention is used, the range of the summation indices being always $\{1,2,3,4\}$.

The conditions (1) and (3) imply that the matrix of $g$ has the form

$$
\left(g_{i j}\right)=\left(\begin{array}{cccc}
A & B & 0 & -B \\
B & A & B & 0 \\
0 & B & A & B \\
-B & 0 & B & A
\end{array}\right),
$$

i.e. it is skew-circulant. Here $A=A(p)$ and $B=B(p)$ are smooth functions of an arbitrary point $p\left(X^{1}, X^{2}, X^{3}, X^{4}\right)$ on $M$. The determinant of $g$ has a value $\operatorname{det}\left(g_{i j}\right)=\left(A^{2}-2 B^{2}\right)^{2}$. It is supposed that

$$
A(p)>\sqrt{2} B(p)>0
$$

43 in order $g$ to be positive definite. A manifold $M$ introduced in this way we denote by $(M, g, S)$.

Now, we consider an associated metric $\tilde{g}$ with $g$, determined by

$$
\tilde{g}(x, y)=g(x, S y)+g(S x, y)
$$

Using (1), (4) and (6) we get that the matrix of its components is

$$
\left(\tilde{g}_{i j}\right)=\left(\begin{array}{cccc}
2 B & A & 0 & -A \\
A & 2 B & A & 0 \\
0 & A & 2 B & A \\
-A & 0 & A & 2 B
\end{array}\right) .
$$


44 Since (5) is valid, it is easy to see that $\tilde{g}$ is an indefinite metric.

The inverse matrices of $\left(g_{i j}\right)$ and $\left(\tilde{g}_{i j}\right)$ are as follows:

$$
\begin{gathered}
\left(g^{i j}\right)=\frac{1}{D}\left(\begin{array}{cccc}
A & -B & 0 & B \\
-B & A & -B & 0 \\
0 & -B & A & -B \\
B & 0 & -B & A
\end{array}\right), \\
\left(\tilde{g}^{i j}\right)=\frac{1}{2 D}\left(\begin{array}{cccc}
-2 B & A & 0 & -A \\
A & -2 B & A & 0 \\
0 & A & -2 B & A \\
-A & 0 & A & -2 B
\end{array}\right),
\end{gathered}
$$

45 where $D=A^{2}-2 B^{2}$.

46 3. Orthogonal $S$-basis of $T_{p} M$

47 If $x$ is a nonzero vector on $(M, g, S)$, then according to (1) we have $S x \neq \pm x$. Therefore the angle $48 \varphi$ between $x$ and $S x$ belongs to the interval $(0, \pi)$. Evidently, the vectors $x, S x, S^{2} x$ and $S^{3} x$ determine 49 six angles, which belong to $(0, \pi)$. For these angles we establish the next statement.

Theorem 1. Let $x$ be a nonzero vector on $(M, g, S)$. Then

$$
\angle(x, S x)=\angle\left(S x, S^{2} x\right)=\angle\left(S^{2} x, S^{3} x\right)=\varphi, \quad \angle\left(x, S^{3} x\right)=\pi-\varphi, \quad \angle\left(x, S^{2} x\right)=\angle\left(S x, S^{3} x\right)=\frac{\pi}{2},
$$

so where $\varphi \in(0, \pi)$.

Proof. Let $x=\left(x^{1}, x^{2}, x^{3}, x^{4}\right)$ be a nonzero vector on $(M, g, S)$. By using (1), we get

$$
S x=\left(x^{2}, x^{3}, x^{4},-x^{1}\right), \quad S^{2} x=\left(x^{3}, x^{4},-x^{1},-x^{2}\right), \quad S^{3} x=\left(x^{4},-x^{1},-x^{2},-x^{3}\right) .
$$

From (2) and (3) it follows

$$
g(x, S x)=-g\left(x, S^{3} x\right), \quad g\left(x, S^{2} x\right)=0 .
$$

Having in mind (4) and (11), we calculate

$$
\begin{array}{r}
g(x, x)=A\left(\left(x^{1}\right)^{2}+\left(x^{2}\right)^{2}+\left(x^{3}\right)^{2}+\left(x^{4}\right)^{2}\right)+2 B\left(x^{1} x^{2}+x^{2} x^{3}+x^{3} x^{4}-x^{1} x^{4}\right), \\
\left.g(x, S x)=A\left(x^{1} x^{2}+x^{2} x^{3}+x^{3} x^{4}-x^{1} x^{4}\right)+B\left(\left(x^{1}\right)^{2}+\left(x^{2}\right)^{2}+\left(x^{3}\right)^{2}+\left(x^{4}\right)^{2}\right)\right) .
\end{array}
$$

Now, due to (3) and (5), we can determine the angle between $x$ and $S x$ and the angle between $x$ and $S^{2} x$ as follows:

$$
\cos \varphi=\frac{g(x, S x)}{g(x, x)}, \quad \cos \phi=\frac{g\left(x, S^{2} x\right)}{g(x, x)} .
$$

We apply (12) and (13) in (14) and find

$$
\begin{gathered}
\cos \varphi=\frac{A\left(x^{1} x^{2}+x^{2} x^{3}+x^{3} x^{4}-x^{1} x^{4}\right)+B\left(\left(x^{1}\right)^{2}+\left(x^{2}\right)^{2}+\left(x^{3}\right)^{2}+\left(x^{4}\right)^{2}\right)}{A\left(\left(x^{1}\right)^{2}+\left(x^{2}\right)^{2}+\left(x^{3}\right)^{2}+\left(x^{4}\right)^{2}\right)+2 B\left(x^{1} x^{2}+x^{2} x^{3}+x^{3} x^{4}-x^{1} x^{4}\right)} \\
\cos \phi=0
\end{gathered}
$$

51 Then, bearing in mind (3) and (12), we get (10). 
52

53

54 The following statements hold.

Theorem 2. Every nonzero vector $x=\left(x^{1}, x^{2}, x^{3}, x^{4}\right)$, which satisfies

$$
\left.4 x^{2} x^{4}\left(\left(x^{1}\right)^{2}-\left(x^{3}\right)^{2}\right)+4 x^{1} x^{3}\left(\left(x^{4}\right)^{2}-\left(x^{2}\right)^{2}\right)+\left(\left(x^{1}\right)^{2}+\left(x^{3}\right)^{2}\right)^{2}+\left(\left(x^{2}\right)^{2}+\left(x^{4}\right)^{2}\right)\right)^{2} \neq 0,
$$

$5 \mathbf{5}$ induces a $S$-basis of $T_{p} M$.

Proof. If a nonzero vector $x \in T_{p} M$ has coordinates $\left(x^{1}, x^{2}, x^{3}, x^{4}\right)$, then using (11) we get the determinant formed by the coordinates of the vectors $x, S x, S^{2} x$ and $S^{3} x$. It is

$$
\left.\triangle=4 x^{2} x^{4}\left(\left(x^{1}\right)^{2}-\left(x^{3}\right)^{2}\right)+4 x^{1} x^{3}\left(\left(x^{4}\right)^{2}-\left(x^{2}\right)^{2}\right)+\left(\left(x^{1}\right)^{2}+\left(x^{3}\right)^{2}\right)^{2}+\left(\left(x^{2}\right)^{2}+\left(x^{4}\right)^{2}\right)\right)^{2} .
$$

56 In case that (15) is valid, we have $\triangle \neq 0$, i.e. $x, S x, S^{2} x$ and $S^{3} x$ form a basis.

Lemma 1. Let a vector $x$ induce a S-basis and let $\varphi$ be the angle between $x$ and $S x$. The following inequalities are valid:

$$
\frac{\pi}{4}<\varphi<\frac{3 \pi}{4}
$$

Proof. We suppose without loss of generality that $g(x, x)=1$. Then, from (3), (12) and (14), we find

$$
g(x, S x)=g\left(S x, S^{2} x\right)=g\left(S^{2} x, S^{3} x\right)=-g\left(x, S^{3} x\right)=\cos \varphi, \quad g\left(x, S^{2} x\right)=g\left(S x, S^{3} x\right)=0
$$

We consider a nonzero vector $y$, such that

$$
y=-\cos \varphi x+S x-\cos \varphi S^{2} x
$$

Since $g$ is a Riemannian metric we have $g(y, y)>0$. Substituting (18) into the latter inequality, and using (17), we get

$$
1-2 \cos ^{2} \varphi>0
$$

57 Then, taking into account $0<\varphi<\pi$, we obtain (16).

58 Bearing in mind Theorem 1, Theorem 2 and Lemma 1, we arrive at the following

59 Theorem 3. For every manifold $(M, g, S)$ an orthogonal $S$-basis of $T_{p} M$ exists.

\section{Almost Einstein manifolds}

Let $\nabla$ be the Riemannian connection of $g$. The curvature tensor $R$ of $\nabla$ is determined by

$$
R(x, y) z=\nabla_{x} \nabla_{y} z-\nabla_{y} \nabla_{x} z-\nabla_{[x, y]} z
$$

The tensor of type $(0,4)$ associated with $R$ is defined by

$$
R(x, y, z, u)=g(R(x, y) z, u)
$$

The Ricci tensor $\rho$ with respect to $g$ is given by the well-known formula

$$
\rho(y, z)=g^{i j} R\left(e_{i}, y, z, e_{j}\right) .
$$


The scalar curvature $\tau$ with respect to $g$ and its associated quantity are determined by

$$
\tau=g^{i j} \rho\left(e_{i}, e_{j}\right), \quad \tau^{*}=\tilde{g}^{i j} \rho\left(e_{i}, e_{j}\right) .
$$

Now, we consider a manifold $(M, g, S)$ with the condition

$$
\nabla S=0
$$

61 i.e., $S$ is a parallel structure with respect to $\nabla$.

Proposition 1. Every manifold $(M, g, S)$ with a parallel structure S satisfies the curvature identity

$$
R(x, y, S z, S u)=R(x, y, z, u) .
$$

Proof. The well-known formula $\left(\nabla_{x} S\right) y=\nabla_{x} S y-S \nabla_{x} y$, together with (23), yields

$$
\nabla_{x} S y=S \nabla_{x} y
$$

On the other hand (19) implies $R(x, y, S z, S u)=g(R(x, y) S z, S u)$. Because of the latter identity, using (3), (19) and (25), we have successively

$$
\begin{aligned}
R(x, y, S z, S u) & =g\left(\nabla_{x} \nabla_{y} S z-\nabla_{y} \nabla_{x} S z-\nabla_{[x, y]} S z, S u\right) \\
& =g\left(\nabla_{x} S\left(\nabla_{y} z\right)-\nabla_{y} S\left(\nabla_{x} z\right)-S\left(\nabla_{[x, y]} z\right), S u\right) \\
& =g\left(S\left(\nabla_{x} \nabla_{y} z-\nabla_{y} \nabla_{x} z-\nabla_{[x, y]} z\right), S u\right)=g(R(x, y) z, u),
\end{aligned}
$$

62 which completes the proof.

Proposition 3. If a manifold $(M, g, S)$ has the property (24), then the components of the Ricci tensor $\rho$ satisfy

$$
\rho_{11}=\rho_{22}=\rho_{33}=\rho_{44}, \quad \rho_{12}=\rho_{23}=\rho_{34}=-\rho_{14}, \quad \rho_{13}=\rho_{24}=0 .
$$


Proof. Due to Proposition 2 the components of the curvature tensor $R$ satisfy (26). For brevity, we denote

$$
R_{1}=R_{1313}, \quad R_{2}=R_{1213} .
$$

Thus, having in mind (8), (21), (26) and (28), we get the components of $\rho$, as follows:

$$
\begin{array}{r}
\rho_{11}=\rho_{22}=\rho_{33}=\rho_{44}=\frac{2}{D}\left(-A R_{1}+2 B R_{2}\right), \\
\rho_{12}=\rho_{23}=\rho_{34}=-\rho_{14}=\frac{2}{D}\left(B R_{1}-A R_{2}\right), \\
\rho_{13}=\rho_{24}=0 .
\end{array}
$$

70

Proof. Due to Proposition 3, for $(M, g, S)$ the equalities (27) are valid. Consequently, from (22), using (8), (9) and (27), we get the values of the scalar curvatures $\tau$ and $\tau^{*}$, as follows:

$$
\tau=\frac{4}{D}\left(A \rho_{11}-2 B \rho_{12}\right), \quad \tau^{*}=\frac{4}{D}\left(-B \rho_{11}+A \rho_{12}\right) .
$$

Immediately from the latter equalities we have

$$
\rho_{11}=\frac{\tau}{4} A+\frac{2 \tau^{*}}{4} B, \quad \rho_{12}=\frac{\tau}{4} B+\frac{\tau^{*}}{4} A,
$$

and bearing in mind (4) and (7) we get

$$
\rho_{11}=\frac{\tau}{4} g_{11}+\frac{\tau^{*}}{4} \tilde{g}_{11}, \quad \rho_{12}=\frac{\tau}{4} g_{12}+\frac{\tau^{*}}{4} \tilde{g}_{12}
$$

Then, taking into account (4), (7), (27) and (32), we obtain

$$
\rho_{i j}=\frac{\tau}{4} g_{i j}+\frac{\tau^{*}}{4} \tilde{g}_{i j}
$$

i.e.

$$
\rho(x, y)=\frac{\tau}{4} g(x, y)+\frac{\tau^{*}}{4} \tilde{g}(x, y)
$$

75 Therefore, comparing (34) with (31), we state that $(M, g, S)$ is an almost Einstein manifold.

Corollary 1. The manifold $(M, g, S)$ with (24) is Einstein if and only if the scalar curvature $\tau^{*}$ vanishes. 
Proof. If $(M, g, S)$ has the scalar curvature which satisfies

$$
\tau^{*}=0,
$$

${ }_{81}(M, g, S)$ by both structures $g$ and $S$.

Theorem 5. Let $(M, g, S)$ have the property (24). Then the curvature tensor $R$ has an expression

$$
R=\frac{\tau}{16}\left(2 \pi_{1}+\pi_{3}\right)+\frac{\tau^{*}}{8} \pi_{2}
$$

where

$$
\begin{aligned}
& \pi_{1}(x, y, z, u)=g(y, z) g(x, u)-g(x, z) g(y, u) \\
& \pi_{2}(x, y, z, u)=g(y, z) \tilde{g}(x, u)+g(x, u) \tilde{g}(y, z)-g(x, z) \tilde{g}(y, u)-g(y, u) \tilde{g}(x, z), \\
& \pi_{3}(x, y, z, u)=\tilde{g}(y, z) \tilde{g}(x, u)-\tilde{g}(x, z) \tilde{g}(y, u) .
\end{aligned}
$$

Proof. Due to Proposition 3, the components of the Ricci tensor $\rho$ of $(M, g, S)$ are given by (29). Therefore, by straightforward computation, we get

$$
R_{1}=-\frac{1}{2}\left(A \rho_{11}+2 B \rho_{12}\right) \quad R_{2}=-\frac{1}{2}\left(B \rho_{11}+A \rho_{12}\right) .
$$

We substitute (32) into the above equalities and obtain

$$
R_{1}=-\frac{1}{8}\left(\left(A^{2}+2 B^{2}\right) \tau+4 A B \tau^{*}\right), \quad R_{2}=-\frac{1}{8}\left(2 A B \tau+\left(2 B^{2}+A^{2}\right) \tau^{*}\right) .
$$

From (4), (7), (28) and (38) it follows

$$
\begin{aligned}
& R_{1313}=\frac{\tau}{16}\left(2\left(g_{13} g_{31}-g_{11} g_{33}\right)+\tilde{g}_{13} \tilde{g}_{31}-\tilde{g}_{11} \tilde{g}_{33}\right)+\frac{\tau^{*}}{8}\left(g_{13} \tilde{g}_{31}+\tilde{g}_{13} g_{31}-\tilde{g}_{11} g_{33}-g_{11} \tilde{g}_{33}\right), \\
& R_{1213}=\frac{\tau}{16}\left(2\left(g_{13} g_{21}-g_{11} g_{23}\right)+\tilde{g}_{13} \tilde{g}_{21}-\tilde{g}_{11} \tilde{g}_{23}\right)+\frac{\tau^{*}}{8}\left(g_{13} \tilde{g}_{21}+\tilde{g}_{13} g_{21}-\tilde{g}_{11} g_{23}-g_{11} \tilde{g}_{23}\right),
\end{aligned}
$$

Consequently, using (4), (7), (26), (28) and (38), we have

$$
R_{i j k h}=\frac{\tau}{16}\left(2\left(g_{i h} g_{j k}-g_{i k} g_{j h}\right)+\tilde{g}_{i h} \tilde{g}_{j k}-\tilde{g}_{i k} \tilde{g}_{j h}\right)+\frac{\tau^{*}}{8}\left(g_{i h} \tilde{g}_{j k}+\tilde{g}_{i h} g_{j k}-\tilde{g}_{i k} g_{j h}-g_{i k} \tilde{g}_{j h}\right),
$$

82 which is equivalent to (36) with (37).

83 5. Curvature properties of $(M, g, S)$

The sectional curvature of a non-degenerate 2-plane $\{x, y\}$ spanned by the vectors $x, y \in T_{p} M$ is the value

$$
k(x, y)=\frac{R(x, y, x, y)}{g(x, x) g(y, y)-g^{2}(x, y)} .
$$

Let $x$ induce a $S$-basis of $T_{p} M$ for $(M, g, S)$ and let $\sigma=\{x, S x\}$ be a 2-plane. Evidently, if $y \in \sigma$ 85 and $y \neq x$, then $S y \notin \sigma$. Consequently, $\sigma$ has only two $S$-bases: $\{x, S x\}$ and $\{-x,-S x\}$. Thus the s6 sectional curvature $k(x, S x)$ depends only on $\varphi=L(x, S x)$. 
Theorem 6. Let $(M, g, S)$ have the property (24) and let a vector $x$ induce a S-basis. Then the sectional curvatures, determined by the S-basis, are

$$
\begin{aligned}
k(x, S x)=k\left(S x, S^{2} x\right)=k\left(x, S^{3} x\right) & =k\left(S^{2} x, S^{3} x\right) \\
& =\frac{1}{16\left(\cos ^{2} \varphi-1\right)}\left(\tau\left(1+2 \cos ^{2} \varphi\right)+4 \tau^{*} \cos \varphi\right), \\
k\left(x, S^{2} x\right)=k\left(S x, S^{3} x\right) & =-\frac{1}{8}\left(\tau\left(1+2 \cos ^{2} \varphi\right)+4 \tau^{*} \cos \varphi\right),
\end{aligned}
$$

87 where $\varphi=L(x, S x)$.

Proof. Let a vector $x$ induce a S-basis. The equalities (3), (12) and (14) imply

$$
\begin{aligned}
g(x, S x) & =g\left(S x, S^{2} x\right)=g\left(S^{2} x, S^{3} x\right)=-g\left(x, S^{3} x\right)=g(x, x) \cos \varphi, \\
g\left(x, S^{2} x\right) & =g\left(S x, S^{3} x\right)=0 .
\end{aligned}
$$

s8 Due to Lemma 1 , the angle $\varphi=L(x, S x)$ satisfies (16).

Now, from (2), (3), (6) and (41) we find

$$
\tilde{g}(x, x)=2 g(x, x) \cos \varphi, \quad \tilde{g}(x, S x)=g(x, x), \quad \tilde{g}\left(x, S^{2} x\right)=0, \quad \tilde{g}\left(x, S^{3} x\right)=-g(x, x)
$$

89 Applying (36), (37), (41) and (42) in (39), we obtain (40).

Corollary 2. Let a vector $x$ induce an orthonormal S-basis. Then

$$
\begin{aligned}
& k(x, S x)=k\left(S x, S^{2} x\right)=k\left(x, S^{3} x\right)=k\left(S^{2} x, S^{3} x\right)=-\frac{\tau}{16} \\
& k\left(x, S^{2} x\right)=k\left(S x, S^{3} x\right)=-\frac{\tau}{8}
\end{aligned}
$$

9o Proof. The proof follows directly from (40), when $\varphi=\frac{\pi}{2}$.

91

Due to Theorem 6 and Corollary 1 we establish the following

Proposition 4. If $(M, g, S)$ with (24) is an Einstein manifold, then the sectional curvatures, determined by an S-basis, are

$$
\begin{aligned}
& k(x, S x)=k\left(S x, S^{2} x\right)=k\left(x, S^{3} x\right)=k\left(S^{2} x, S^{3} x\right)=\frac{\tau\left(1+2 \cos ^{2} \varphi\right)}{16\left(\cos ^{2} \varphi-1\right)} \\
& k\left(x, S^{2} x\right)=k\left(S x, S^{3} x\right)=-\frac{\tau}{8}\left(1+2 \cos ^{2} \varphi\right) .
\end{aligned}
$$

Now, we recall that the Ricci curvature in the direction of a non-zero vector $x$ is the value

$$
r(x)=\frac{\rho(x, x)}{g(x, x)} .
$$

Theorem 7. Let $(M, g, S)$ have the property (24) and let a vector $x$ induce a S-basis. Then the Ricci curvatures are

$$
r(x)=r(S x)=r\left(S^{2} x\right)=r\left(S^{3} x\right)=\frac{\tau}{4}+\frac{\tau^{*}}{2} \cos \varphi,
$$

92 where $\varphi=\angle(x, S x)$. 
Proof. According to Theorem 4, the Ricci tensor $\rho$ is given by (34). Then, using (3), we find

$$
\rho(x, x)=\rho(S x, S x)=\rho\left(S^{2} x, S^{2} x\right)=\rho\left(S^{3} x, S^{3} x\right)=\frac{\tau}{4} g(x, x)+\frac{\tau^{*}}{4} \tilde{g}(x, x) .
$$

93 Let a vector $x$ induce a $S$-basis. From (3), (42), (43) and (45) it follows (44).

Further, Theorem 7 and Corollary 1 imply the next statement.

Proposition 5. Let $(M, g, S)$ with (24) be an Einstein manifold. Then the Ricci curvatures are

$$
r(x)=r(S x)=r\left(S^{2} x\right)=r\left(S^{3} x\right)=\frac{\tau}{4} .
$$

95 Proof. These equalities follow directly by substituting $\tau^{*}=0$ into (44).

96 6. Manifolds with parallel structures

In this section we study a manifold $(M, g, S)$, whose structure $S$ satisfies (23). Also, we consider an associated manifold $(M, g, J)$ with a structure $J=S^{2}$. Bearing in mind (2) and (3), we get that the manifold $(M, g, J)$ is almost Hermitian and the structure $J$ is almost complex. In case that $J$ is parallel $(M, g, J)$ is a Kähler manifold. The characteristic condition of a Kähler manifold is

$$
\nabla J=0
$$

97 We note that equalities (23) and $J=S^{2}$ imply (46).

Theorem 8. Let $(M, g, S)$ have the property (23). Then the scalar curvatures $\tau$ and $\tau^{*}$ satisfy

$$
3 \tau_{1}=\tau_{2}^{*}-\tau_{4}^{*}, \quad 3 \tau_{2}=\tau_{1}^{*}+\tau_{3}^{*}, \quad 3 \tau_{3}=\tau_{2}^{*}+\tau_{4}^{*}, \quad 3 \tau_{4}=-\tau_{1}^{*}+\tau_{3}^{*},
$$

98 where $\tau_{i}=\frac{\partial \tau}{\partial X^{i}}, \tau_{i}^{*}=\frac{\partial \tau^{*}}{\partial X^{i}}$.

Proof. It is known that in a Riemannian manifold for the scalar curvature $\tau$ and the Ricci tensor $\rho$ it is valid

$$
\nabla_{i} \rho_{k}^{i}=\frac{1}{2} \nabla_{k} \tau
$$

99 where $\rho_{k}^{i}=\rho_{a k} g^{a i}$.

On the other hand, if $(M, g, S)$ satisfies (23), then it satisfies (24). Therefore, the Ricci tensor has the expression (33). Hence, from (1), (4), (7), (8) and (33), we get

$$
\rho_{k}^{i}=\frac{\tau}{4} \delta_{k}^{i}+\frac{\tau^{*}}{4}\left(S_{k}^{i}-\left(S_{k}^{i}\right)^{3}\right)
$$

where $\delta_{k}^{i}$ are the Kronecker symbols. Using the above equalities, (23) and (48) we obtain

$$
\tau_{k}=\frac{\tau_{i}}{4} \delta_{k}^{i}+\frac{\tau_{i}^{*}}{4}\left(S_{k}^{i}-\left(S_{k}^{i}\right)^{3}\right)
$$

Then, from (1) it follows (47).

According to Theorem 8 and Corollary 1 we establish the following

Proposition 6. If $(M, g, S)$ with (23) is an Einstein manifold, then the scalar curvature $\tau$ is a constant. 
Theorem 9. The manifold $(M, g, S)$ satisfies (23) if and only if

$$
A_{1}=B_{2}-B_{4}, \quad A_{2}=B_{1}+B_{3}, \quad A_{3}=B_{2}+B_{4}, \quad A_{4}=B_{4}=B_{3}-B_{1},
$$

104 where $A_{i}=\frac{\partial A}{\partial X^{i}}, B_{i}=\frac{\partial B}{\partial X^{i}}$.

Proof. If $\Gamma_{i j}^{s}$ are the Christoffel symbols of $\nabla$, then

$$
\nabla_{i} S_{j}^{t}=\partial_{i} S_{j}^{t}+\Gamma_{i k}^{t} S_{j}^{k}-\Gamma_{i j}^{k} S_{k}^{t}
$$

Together with (23), (50) yields

$$
\Gamma_{i k}^{t} S_{j}^{k}=\Gamma_{i j}^{k} S_{k}^{t}
$$

From (1) and (51) we get

$$
\begin{aligned}
& \Gamma_{11}^{1}=\Gamma_{12}^{2}=\Gamma_{13}^{3}=\Gamma_{14}^{4}=\Gamma_{22}^{3}=\Gamma_{23}^{4}=-\Gamma_{24}^{1}=-\Gamma_{33}^{1}=-\Gamma_{34}^{2}=-\Gamma_{44}^{3}, \\
& \Gamma_{11}^{2}=\Gamma_{12}^{3}=\Gamma_{13}^{4}=-\Gamma_{14}^{1}=\Gamma_{22}^{4}=-\Gamma_{23}^{1}=-\Gamma_{24}^{2}=-\Gamma_{33}^{2}=-\Gamma_{34}^{3}=-\Gamma_{44}^{4} \\
& \Gamma_{11}^{3}=\Gamma_{12}^{4}=-\Gamma_{13}^{1}=-\Gamma_{14}^{2}=-\Gamma_{22}^{1}=-\Gamma_{23}^{2}=-\Gamma_{24}^{3}=-\Gamma_{33}^{3}=-\Gamma_{34}^{4}=\Gamma_{44}^{1} \\
& \Gamma_{11}^{4}=-\Gamma_{12}^{1}=-\Gamma_{13}^{2}=-\Gamma_{14}^{3}=-\Gamma_{22}^{2}=-\Gamma_{23}^{3}=-\Gamma_{24}^{4}=-\Gamma_{33}^{4}=\Gamma_{34}^{1}=\Gamma_{44}^{2} .
\end{aligned}
$$

Now, using (1), (4), (8) and the well known identities

$$
2 \Gamma_{i j}^{s}=g^{a s}\left(\partial_{i} g_{a j}+\partial_{j} g_{a i}-\partial_{a} g_{i j}\right)
$$

we calculate

$$
\begin{aligned}
& \Gamma_{11}^{1}=\frac{1}{2 D}\left(A A_{1}-B\left(4 B_{1}-A_{2}+A_{4}\right)\right), \\
& \Gamma_{11}^{3}=\frac{1}{2 D}\left(B\left(A_{2}+A_{4}\right)-A A_{3}\right) \text {, } \\
& \Gamma_{12}^{1}=\frac{1}{2 D}\left(A A_{2}-B\left(A_{1}+B_{2}+B_{4}\right)\right) \text {, } \\
& \Gamma_{11}^{2}=\frac{1}{2 D}\left(A\left(2 B_{1}-A_{2}\right)+B\left(A_{3}-A_{1}\right)\right), \\
& \Gamma_{11}^{4}=\frac{1}{2 D}\left(B\left(A_{1}+A_{3}\right)-A\left(2 B_{1}+A_{4}\right)\right) \text {, } \\
& \Gamma_{12}^{3}=\frac{1}{2 D}\left(A\left(B_{1}-B_{3}\right)-B\left(A_{1}-B_{2}-B_{4}\right)\right) \text {, } \\
& \Gamma_{13}^{1}=\frac{1}{2 D}\left(A A_{3}-2 B B_{3}\right) \text { ， } \\
& \Gamma_{13}^{3}=\frac{1}{2 D}\left(A A_{1}-2 B B_{1}\right) \text {, } \\
& \Gamma_{14}^{1}=\frac{1}{2 D}\left(A A_{4}+B\left(A_{1}-B_{2}-B_{4}\right)\right) ， \\
& \Gamma_{12}^{2}=\frac{1}{2 D}\left(A A_{1}-B\left(A_{2}+B_{1}-B_{3}\right)\right), \\
& \Gamma_{12}^{4}=\frac{1}{2 D}\left(B\left(A_{2}+B_{3}-B_{1}\right)-A\left(B_{2}+B_{4}\right)\right) \text {, } \\
& \Gamma_{13}^{2}=\frac{1}{2 D}\left(A\left(B_{1}+B_{3}\right)-B\left(A_{1}+A_{3}\right)\right) \text {, } \\
& \Gamma_{14}^{3}=\frac{1}{2 D}\left(A\left(B_{1}+B_{3}\right)-B\left(A_{1}+B_{2}+B_{4}\right)\right) \text {, } \\
& \Gamma_{13}^{4}=\frac{1}{2 D}\left(A\left(B_{1}-B_{3}\right)+B\left(A_{3}-A_{1}\right)\right) \text {, } \\
& \Gamma_{14}^{2}=\frac{1}{2 D}\left(A\left(B_{2}+B_{4}\right)-B\left(A_{4}+B_{1}+B_{3}\right)\right) \text {, } \\
& \Gamma_{22}^{1}=\frac{1}{2 D}\left(A\left(2 B_{2}-A_{1}\right)-B\left(A_{2}+A_{4}\right)\right) \text {, } \\
& \Gamma_{14}^{4}=\frac{1}{2 D}\left(A A_{1}+B\left(A_{4}-B_{1}-B_{3}\right)\right), \\
& \Gamma_{22}^{3}=\frac{1}{2 D}\left(A\left(2 B_{2}-A_{3}\right)+B\left(A_{4}-A_{2}\right)\right) \text {, } \\
& \Gamma_{22}^{2}=\frac{1}{2 D}\left(A A_{2}-B\left(4 B_{2}-A_{1}-A_{3}\right)\right) \text {, } \\
& \Gamma_{23}^{1}=\frac{1}{2 D}\left(A\left(B_{3}-B_{1}\right)-B\left(A_{3}-B_{2}+B_{4}\right)\right) \text {, } \\
& \Gamma_{22}^{4}=\frac{1}{2 D}\left(B\left(A_{3}-A_{1}\right)-A A_{4}\right), \\
& \Gamma_{23}^{2}=\frac{1}{2 D}\left(A A_{3}+B\left(B_{1}-B_{3}-A_{2}\right)\right) \text {, } \\
& \Gamma_{23}^{3}=\frac{1}{2 D}\left(A A_{2}-B\left(B_{2}-B_{4}+A_{3}\right)\right) \text {, } \\
& \Gamma_{24}^{1}=\frac{1}{2 D}\left(A\left(B_{4}-B_{2}\right)-B\left(A_{4}-A_{2}\right)\right) \text {, } \\
& \Gamma_{23}^{4}=\frac{1}{2 D}\left(A\left(B_{2}-B_{4}\right)-B\left(A_{2}+B_{1}-B_{3}\right)\right) \text {, } \\
& \Gamma_{24}^{3}=\frac{1}{2 D}\left(A\left(B_{2}+B_{4}\right)-B\left(A_{2}+A_{4}\right)\right) \text {, } \\
& \Gamma_{24}^{2}=\frac{1}{2 D}\left(A A_{4}-2 B B_{4}\right) \text {, } \\
& \Gamma_{33}^{1}=\frac{1}{2 D}\left(B\left(A_{2}-A_{4}\right)-A A_{1}\right) \text {, } \\
& \Gamma_{33}^{3}=\frac{1}{2 D}\left(A A_{3}-B\left(4 B_{3}-A_{2}-A_{4}\right)\right) \text {, } \\
& \Gamma_{34}^{1}=\frac{1}{2 D}\left(B\left(A_{3}+B_{2}-B_{4}\right)-A\left(B_{1}+B_{3}\right)\right) \text {, } \\
& \Gamma_{34}^{3}=\frac{1}{2 D}\left(A B_{4}-B\left(A_{3}-B_{2}+B_{4}\right)\right) \text {, } \\
& \Gamma_{44}^{1}=\frac{1}{2 D}\left(B\left(A_{2}+A_{4}\right)-A\left(2 B_{4}+A_{1}\right)\right) \text {, } \\
& \Gamma_{44}^{3}=\frac{1}{2 D}\left(A\left(2 B_{4}-A_{3}\right)+B\left(A_{2}-A_{4}\right)\right) \text {, } \\
& \Gamma_{24}^{4}=\frac{1}{2 D}\left(A A_{2}-2 B B_{2}\right) \text { ， } \\
& \Gamma_{33}^{2}=\frac{1}{2 D}\left(A\left(2 B_{3}-A_{2}\right)+B\left(A_{1}-A_{3}\right)\right) \text {, } \\
& \Gamma_{33}^{4}=\frac{1}{2 D}\left(A\left(2 B_{3}-A_{4}\right)-B\left(A_{1}+A_{3}\right)\right) \text {, } \\
& \Gamma_{34}^{2}=\frac{1}{2 D}\left(A\left(B_{4}-B_{2}\right)+B\left(B_{3}+B_{1}-B_{4}\right)\right) \text {, } \\
& \Gamma_{34}^{4}=\frac{1}{2 D}\left(A A_{3}-B\left(B_{1}+B_{3}+B_{4}\right)\right) \text {, } \\
& \Gamma_{44}^{2}=\frac{1}{2 D}\left(B\left(A_{1}+A_{3}\right)-A A_{2}\right) \text {, } \\
& \Gamma_{44}^{4}=\frac{1}{2 D}\left(A A_{4}-B\left(4 B_{4}+A_{1}-A_{3}\right)\right) \text {. }
\end{aligned}
$$

We apply (53) in (52) and obtain the conditions (49).

Vice versa. Let (49) hold true. We put equalities (49) into (53) and find (52). Hence (1) and (52) imply (51). Consequently, from (1), (50) and (51) we get (23). 
108

Theorem 10. The manifold $(M, g, J)$ is Kähler if and only if the equalities (49) are valid.

Proof. Having in mind (1), we get that the components of the structure $J=S^{2}$ on $(M, g, J)$ are given by the skew-circulant matrix

$$
\left(J_{j}^{k}\right)=\left(\begin{array}{cccc}
0 & 0 & 1 & 0 \\
0 & 0 & 0 & 1 \\
-1 & 0 & 0 & 0 \\
0 & -1 & 0 & 0
\end{array}\right)
$$

Therefore, from (46), (54) and

$$
\nabla_{i} J_{j}^{t}=\partial_{i} J_{j}^{t}+\Gamma_{i k}^{t} J_{j}^{k}-\Gamma_{i j}^{k} J_{k}^{t}
$$

it follows

$$
\Gamma_{i k}^{t} J_{j}^{k}=\Gamma_{i j}^{k} J_{k}^{t}
$$

Together with (54), (55) yields

$$
\begin{array}{lll}
\Gamma_{11}^{1}=\Gamma_{13}^{3}=-\Gamma_{33}^{1}, & \Gamma_{14}^{4}=\Gamma_{23}^{4}=\Gamma_{12}^{2}=-\Gamma_{34^{\prime}}^{2} & \Gamma_{22}^{3}=-\Gamma_{24}^{1}=-\Gamma_{44^{\prime}}^{3} \\
\Gamma_{11}^{2}=\Gamma_{13}^{4}=-\Gamma_{33}^{2}, & \Gamma_{14}^{1}=\Gamma_{23}^{1}=-\Gamma_{12}^{3}=\Gamma_{34^{\prime}}^{3} & \Gamma_{22}^{4}=-\Gamma_{24}^{2}=-\Gamma_{44}^{4} \\
\Gamma_{11}^{3}=-\Gamma_{13}^{1}=-\Gamma_{33}^{3}, & \Gamma_{14}^{2}=\Gamma_{23}^{2}=-\Gamma_{12}^{4}=\Gamma_{34}^{4}, & \Gamma_{22}^{1}=\Gamma_{24}^{3}=-\Gamma_{44^{\prime}}^{1} \\
\Gamma_{11}^{4}=-\Gamma_{13}^{2}=-\Gamma_{33^{\prime}}^{4} & \Gamma_{14}^{3}=\Gamma_{23}^{3}=\Gamma_{12}^{1}=-\Gamma_{34^{\prime}}^{1} & \Gamma_{22}^{2}=\Gamma_{24}^{4}=-\Gamma_{44}^{2} .
\end{array}
$$

We apply (53) in (56) and obtain conditions (49).

Vice versa. From (49) it follows (23). Obviously (23) implies (46).

Bearing in mind Theorem 9 and Theorem 10 we state the following

Corollary 3. The structure $S$ of $(M, g, S)$ is parallel with respect to $\nabla$ if and only if the structure $J$ of $(M, g, J)$ is parallel with respect to $\nabla$.

\section{Lie groups as 4-dimensional Riemannian manifolds with skew-circulant structures}

Let $G$ be a 4-dimensional real connected Lie group and $\mathbf{g}$ be its Lie algebra with a basis $\left\{x_{1}, x_{2}, x_{3}, x_{4}\right\}$. We introduce a structure $S$ and left invariant metric $g$ as follows

$$
\begin{gathered}
S x_{1}=x_{2}, S x_{2}=x_{3}, S x_{3}=x_{4}, S x_{4}=-x_{1}, \\
g\left(x_{i}, x_{j}\right)= \begin{cases}0, & i \neq j \\
1, & i=j .\end{cases}
\end{gathered}
$$

Obviously (2) and (3) are valid. Therefore $(G, g, S)$ is a Riemannian manifold of the considered type.

For the manifold $(G, g, S)$ we suppose that $S$ is an Abelian structure, i.e.

$$
\left[x_{i}, x_{j}\right]=\left[S x_{i}, S x_{j}\right]
$$

According to (57), (59) and the Jacobi identity for the commutators $\left[x_{i}, x_{j}\right]$ we obtain

$$
\begin{aligned}
& {\left[x_{1}, x_{2}\right]=\left[x_{1}, x_{4}\right]=\left[x_{2}, x_{3}\right]=\left[x_{3}, x_{4}\right]=\lambda_{1} x_{1}+\lambda_{2} x_{2}+\lambda_{3} x_{3}+\lambda_{4} x_{4},} \\
& {\left[x_{1}, x_{3}\right]=\left[x_{2}, x_{4}\right]=\left(\lambda_{2}-\lambda_{4}\right) x_{1}+\left(\lambda_{1}+\lambda_{3}\right) x_{2}+\left(\lambda_{2}+\lambda_{4}\right) x_{3}+\left(\lambda_{3}-\lambda_{1}\right) x_{4}}
\end{aligned}
$$

where $\lambda_{i} \in \mathbb{R}$.

It is easy to see that a manifold $(G, g, S)$ with a Lie algebra $\mathbf{g}$, determined by (60), has an Abelian structure $S$. 

tensor

$$
\begin{aligned}
& R_{1313}=R_{2424}=R_{1324}=2 R_{1212}=2 R_{1414}=2 R_{2323}=2 R_{3434}=2 R_{1223}=2 R_{1214} \\
& =2 R_{1434}=2 R_{1234}=2 R_{2334}=2 R_{2314}=2\left(\lambda_{1}^{2}+\lambda_{2}^{2}+\lambda_{3}^{2}+\lambda_{4}^{2}\right), \\
& R_{1213}=R_{1224}=R_{1413}=R_{2414}=R_{2423}=R_{2313}=R_{1334}=R_{2434} \\
& =2\left(\lambda_{1} \lambda_{2}+\lambda_{2} \lambda_{3}+\lambda_{3} \lambda_{4}-\lambda_{1} \lambda_{4}\right) \text {. }
\end{aligned}
$$

The rest of the nonzero components are obtained from the properties

$$
R_{i j k s}=R_{k s i j}, R_{i j k s}=-R_{j i k s}=-R_{i j k k} .
$$

From (58), (62) and the formula (21) we get the components of the Ricci tensor $\rho$ :

$$
\begin{array}{r}
\rho_{11}=\rho_{22}=\rho_{33}=\rho_{44}=-4\left(\lambda_{1}^{2}+\lambda_{2}^{2}+\lambda_{3}^{2}+\lambda_{4}^{2}\right), \\
\rho_{12}=\rho_{23}=\rho_{34}=-4\left(\lambda_{1} \lambda_{2}+\lambda_{2} \lambda_{3}+\lambda_{3} \lambda_{4}-\lambda_{1} \lambda_{4}\right), \\
\rho_{13}=\rho_{24}=0, \quad \rho_{14}=-\rho_{12} .
\end{array}
$$

Now, using (6) and (58), we find the components of $\tilde{g}$ and the components of its inverse. They are as follows:

$$
\begin{array}{lll}
\tilde{g}_{11}=\tilde{g}_{22}=\tilde{g}_{33}=\tilde{g}_{44}=0, & \tilde{g}_{12}=\tilde{g}_{23}=\tilde{g}_{34}=-\tilde{g}_{14}=1, & \tilde{g}_{13}=\tilde{g}_{24}=0, \\
\tilde{g}^{11}=\tilde{g}^{22}=\tilde{g}^{33}=\tilde{g}^{44}=0, & \tilde{g}^{12}=\tilde{g}^{23}=\tilde{g}^{34}=-\tilde{g}^{14}=\frac{1}{2}, & \tilde{g}^{13}=\tilde{g}^{24}=0 .
\end{array}
$$

Then, applying (58), (63) in (22), we get the values of the scalar curvatures $\tau$ and $\tau^{*}$ as follows:

$$
\tau=-16\left(\lambda_{1}^{2}+\lambda_{2}^{2}+\lambda_{3}^{2}+\lambda_{4}^{2}\right), \quad \tau^{*}=-16\left(\lambda_{1} \lambda_{2}+\lambda_{2} \lambda_{3}+\lambda_{3} \lambda_{4}-\lambda_{1} \lambda_{4}\right) .
$$

Consequently, the equalities (58), (63) and (64) imply (33), i.e. $(G, g, S)$ is an almost Einstein manifold.

Further, using (39), (58) and (62), for the sectional curvatures of the basic 2-planes we find

$$
\begin{array}{r}
k\left(x_{2}, x_{4}\right)=k\left(x_{1}, x_{3}\right)=2\left(\lambda_{1}^{2}+\lambda_{2}^{2}+\lambda_{3}^{2}+\lambda_{4}^{2}\right), \\
k\left(x_{1}, x_{2}\right)=k\left(x_{1}, x_{4}\right)=k\left(x_{2}, x_{3}\right)=k\left(x_{3}, x_{4}\right)=\lambda_{1}^{2}+\lambda_{2}^{2}+\lambda_{3}^{2}+\lambda_{4}^{2} .
\end{array}
$$


(i) The nonzero components of the Ricci tensor $\rho$ are

$$
\rho_{11}=\rho_{22}=\rho_{33}=\rho_{44}=-12\left(\lambda_{2}^{2}+\lambda_{4}^{2}\right) ;
$$

(ii) The manifold is Einstein and the scalar curvatures $\tau$ and $\tau^{*}$ are

$$
\tau=-48\left(\lambda_{2}^{2}+\lambda_{4}^{2}\right), \quad \tau^{*}=0
$$

(iii) The sectional curvatures of the basic 2-planes are

$$
k\left(x_{2}, x_{4}\right)=k\left(x_{1}, x_{3}\right)=6\left(\lambda_{2}^{2}+\lambda_{4}^{2}\right), \quad k\left(x_{1}, x_{2}\right)=k\left(x_{1}, x_{4}\right)=k\left(x_{2}, x_{3}\right)=k\left(x_{3}, x_{4}\right)=3\left(\lambda_{2}^{2}+\lambda_{4}^{2}\right) .
$$

Proof. We put $\lambda_{1}=\lambda_{2}+\lambda_{4}$ and $\lambda_{3}=\lambda_{4}-\lambda_{2}$ into each of the equalities (60), (63), (64) and (65). 
Author Contributions: All authors contributed equally to this work.

Funding: This work is partially supported by project MU19-FMI-020 of the Scientific Research Fund, Paisii Hilendarski University of Plovdiv, Bulgaria.

Acknowledgments: The authors are grateful to Professor Dr. G. Dzhelepov for his valuable comments on this paper.

Conflicts of Interest: The authors declare no conflict of interest.

\section{References}

1. Davis, P. J. Circulant matrices, A Wiley-Interscience Publication. Pure and Applied Mathematics. John Wiley and Sons, New York-Chichester-Brisbane, New York, USA, 1979; pp. 250.

2. Dzhelepov G.; Dokuzova I.; Razpopov D. On a three-dimensional Riemannian manifold with an additional structure, Plovdiv Univ. Paisiu Khilendarski Nauchn. Trud. Mat., 2011, 38 (3), 17-27.

3. Gray, R. M. Toeplitz and circulant matrices: A review, Found. Trends Commun. Inf. Theory 2006, 2 (3), 155-239.

4. Gray, A. Curvature identities for Hermitian and almost Hermitian manifolds, Tôhoku Math. J. (2) 1976, 28 (4), 601-612.

5. Gray, A.; Hervella, L.M. The sixteen classes of almost Hermitian manifolds and their linear invariants, Ann. Mat. Pura Appl. (4) 1980, 123, 35-58.

6. Gray, A.; Vanhecke, L. Almost Hermitian manifolds with constant holomorphic sectional curvatures, Časopis Pést. Mat. 1979, 104 (2), 170-179.

7. Jiang, XY.; Hong, K. Explicit determinants of the k-Fibonacci and k-Lucas RSFPLR circulant matrix in codes. In: Yang Y., Ma M., Liu B. (eds) Information Computing and Applications. ICICA 2013. Communications in Computer and Information Science, 391. Springer, Berlin, Heidelberg, Germany 2013; 625-637.

8. Liu, K.; Yang, X. Ricci curvatures on Hermitian manifolds, Trans. American Math. Soc. 2017, 369 (7), 5157-5196.

9. Muzychuk, M. A Solution of the isomorphism problem for circulant graphs, In: Proc. London Math. Soc. (3) 2004, 88 (1), 1-41.

10. Matsuo, K. Pseudo-Bochner curvature tensor on Hermitian manifolds, Colloq. Math. 1999, 80 (2), 201-209.

11. Olson, B.; Shaw, S.; Shi, C.; Pierre C.; Parker, R. G. Circulant matrices and their application to vibration analysis, Appl. Mech. Rev., 2014, 66 (4), 1-41.

12. Razpopov, D. Four-dimensional Riemannian manifolds with two circulant structures, Math. Educ. Math. 2015, 44, In: Proc. of 44-th Spring Conf. of UBM, SOK Kamchia, Bulgaria, 179-185.

13. Roth, R. M.; Lempel, A. Application of circulant matrices to the construction and decoding of linear codes, IEEE Trans. Inform. Theory, 1990, 36 (5), 1157-1163.

14. Prvanović, M. Conformally invariant tensors of an almost Hermitian manifold associated with the holomorphic curvature tensor, J. Geom. 2012, 103 (1), 89-101.

15. Vanhecke, L. Some almost Hermitian manifolds with constant holomorphic sectional curvature, J. Differential. Geom. 1977, 12(4): 461-471.

16. Yang, B.; Zheng, F. On curvature tensor of Hermitian manifolds, Comm. Anal. Geom.2018, 26 (5), 1195-1222.

17. Yano K. Differential geometry on complex and almost complex spaces, International Series of Monographs in Pure and Applied Mathematics 49, A Pergamon Press Book The Macmillan and Co., New York, USA, 1965; pp. 326. 